\title{
Unresectable Dedifferentiated Liposarcoma
}

National Cancer Institute

\section{Source}

National Cancer Institute. Unresectable Dedifferentiated Liposarcoma. NCI Thesaurus.

Code C150598.

Dedifferentiated liposarcoma that is not amenable to surgical resection. 